\title{
Laminar flow of a viscoelastic shear-thinning liquid through a plane sudden expansion preceded by a gradual contraction
}

\author{
By R. J. Poole ${ }^{1}$, M. P. Escudier ${ }^{1}$ and P. J. Oliveira ${ }^{2}$ \\ ${ }^{1}$ Department of Engineering, Mechanical Engineering, University of Liverpool, \\ Brownlow Hill, Liverpool L69 3GH, UK \\ (robpoole@liv.ac.uk) \\ ${ }^{2}$ Departmento Engenharia Electromecanica, Universidade da Beira Interior, \\ 6200 Covilha, Portugal
}

Experimental observations are reported for the laminar flow of a viscoelastic liquid through a symmetrical plane sudden expansion preceded by a gradual contraction from a square duct. As is well known, for Newtonian fluid flow above a critical Reynolds number the flowfield downstream of an expansion becomes asymmetric. For the viscoelastic liquid investigated here the asymmetry is greatly reduced, with very similar reattachment lengths for the two recirculation regions. More significantly, the flow unexpectedly develops a strongly three-dimensional jet-like structure, with side-to-side symmetry centred on the 'vertical' symmetry plane of the contraction/expansion geometry. Especially interesting is the flow within the contraction itself, where the nature of the flow field for the viscoelastic liquid is also fundamentally different to that for a comparable Newtonian fluid flow: large velocity overshoots with very strong gradients occur near to the sidewalls that, due to their appearance, we have termed 'cat's ears'. The fully developed approach flow in the square duct is unremarkable.

Keywords: viscoelastic; non-Newtonian; sudden expansion; gradual contraction

\section{Introduction}

For laminar flow of a Newtonian fluid through a plane sudden expansion, it is well known that above a critical Reynolds number when the expansion ratio $R=D / d$ exceeds 1.5 the flowfield downstream of the expansion develops top-tobottom asymmetry, if the rectangular inlet to the expansion is regarded as having a horizontal orientation. This asymmetry has been observed both experimentally (e.g. Durst et al. 1974; Cherdron et al. 1978; Fearn et al. 1990) and numerically (e.g. Drikakis 1997; Schrek \& Schafer 2000). The critical Reynolds number $\left(\mathrm{Re}_{\mathrm{CR}}\right)$ at which this switch to asymmetric flow is observed is dependent upon both the upstream flow conditions, e.g. fully developed or uniform velocity profile, and the aspect ratio of the expansion, $A_{d}=$ duct width/ duct height at inlet $=w / d$ or $A_{h}=\operatorname{duct}$ width $/$ step height $=w / h($ table 1$)$.

In contrast to the situation for a Newtonian fluid, the laminar flow of nonNewtonian, viscoelastic, liquids through sudden expansions has received scant 
Table 1. Notation

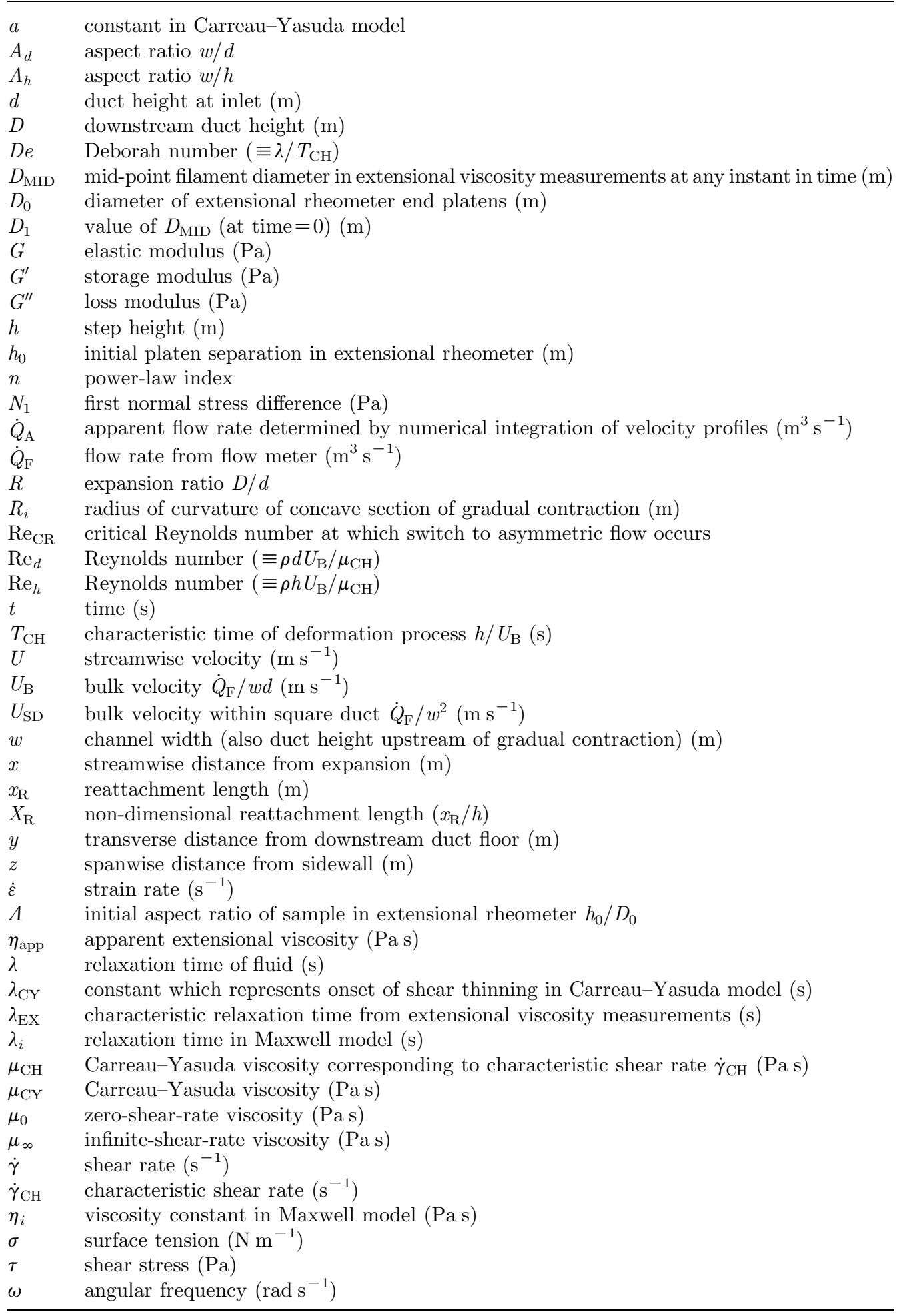


attention in the literature and is largely restricted to a handful of papers at low Reynolds numbers, where the flow remains symmetric, and has involved flow visualization and theoretical modelling but no detailed measurements of the flow field.

The first to investigate expansive flows of viscoelastic, shear-thinning liquids were Halmos \& Boger (1976) who investigated the flow of polyacrylamide (PAA) solutions through a 2:1 expansion for generalized Reynolds numbers $\left(=\rho U_{\mathrm{B}}^{2-n} d^{n} / k\right)$ in the range $1-100$. Streak photography was used for flow visualization while a flash technique was used to obtain centreline velocity estimates. In earlier work, (Halmos et al. 1975a,b), these authors presented a numerical solution with experimental verification for the flow of inelastic, powerlaw fluids through an axisymmetric sudden expansion. Halmos \& Boger (1976) concluded that as a viscoelastic fluid flows through a sudden expansion it releases some of its stored energy, resulting in an expansion of the main flow and compression of the secondary cell (the recirculation region).

Townsend \& Walters (1994) also used flow visualization to observe the flowfield downstream of both a two-dimensional and a three-dimensional expansion for a $0.15 \%$ aqueous solution of PAA. Although these authors do not provide Reynolds numbers for the polymer-solution experiments, the 'comparison' simulation results are for a Reynolds number $\left(=\rho U_{\mathrm{B}} d / \mu\right)$ of 5 . The conclusion drawn from their study was that the viscoelasticity of this polymer solution damped out the large vortex activity (which is generated by fluid inertia; Townsend \& Walters 1994) and caused any recirculating fluid to be pushed into the corners of the expansion. A theoretical model employed to simulate the flow field numerically produced results in good physical agreement with the flow visualization but these were essentially qualitative in nature due to the lack of quantitative rheological and velocity data.

Darwish et al. (1992) and later Missirlis et al. (1998) used a finite-volume technique to simulate the flow of a viscoelastic liquid through a $4: 1$ plane sudden expansion at a Reynolds number $\left(=\rho U_{\mathrm{B}} d / \mu\right)$ of 0.2 . In both studies it was assumed that the flow is two-dimensional but in neither was the work validated by comparison with experimental data. Instead, verification was inferred using grid refinement to obtain grid-independent results. Missirlis et al. showed that the suppression of vortex activity is related to the Deborah number $D e=\lambda / T_{\mathrm{CH}}$, where $T_{\mathrm{CH}}$ is a characteristic time of the deformation process being observed and $\lambda$ is a characteristic time of the material. They showed that as $D e$ is increased beyond a critical value of 3 the recirculation zone is completely eliminated.

The experiments of Townsend \& Walters (1994) were also used as the basis for comparison in the numerical simulation work of Baloch et al. (1996) who modelled expansion flows in two- and three-dimensions using the class of constitutive models proposed by Phan-Thien \& Tanner (1977) at Reynolds numbers $\left(=\rho U_{\mathrm{B}} d / \mu\right.$, where $\mu$ is the sum of the solvent and polymeric viscosity) of 2 and 4. Once again good qualitative agreement with the experimental visualizations was found and the conclusion again drawn that viscoelasticity suppresses vortex activity and that this suppression is linked to the phenomenon of die swell.

There is significant renewed interest in sudden-expansion flows and three works have, for the first time, investigated non-Newtonian fluid flow though a plane sudden expansion, of expansion ratio $R=3$, at sufficiently high Reynolds 
numbers (ca 100) for asymmetric flow to occur. Neofytou \& Drikakis (2003) simulated the flow of blood using the Casson, power-law and Quemada viscosity models. All three models are inelastic and represent only the shear-thinning behaviour of blood. They found that the top-to-bottom asymmetry also occurs for the flow of these shear-thinning fluids and that the critical Reynolds number is dependent on the viscosity-model parameters. The effect of shear thinning alone has a negligible effect on the critical Reynolds number, whereas the models incorporating a 'yield stress' exhibited an increase in the critical Re, up to a factor of 2 compared to the Newtonian value, with increasing values of the yieldstress parameter (i.e. Bingham number). Manica \& De Bortoli (2004) essentially confirmed these findings for the flow of power-law fluids. Viscoelastic effects, such as extensional viscosity variations and those associated with normal-stress differences, are not modelled in either of these studies. In contrast, Oliveira (2003) attempted to incorporate viscoelastic effects in the flow of a nonNewtonian liquid by employing a modified finite extensionability constitutive equation (FENE-CR). Here again, asymmetric flow was predicted to occur above a critical Reynolds number $\left(\operatorname{Re}_{\mathrm{CR}}\right)$, which was dependent upon the concentration and extensionability parameters chosen for the model. These calculations showed that the trend is for $\operatorname{Re}_{\mathrm{CR}}$ always to increase beyond the Newtonian value, i.e. the effect of viscoelasticity is a stabilizing one. All of these studies are purely numerical in nature and had to rely for validation on grid independency or agreement with simulations for the flow of a Newtonian fluid, a course of action that is clearly not ideal and something the current work aims to address by providing detailed rheological and velocity data.

In discussing our observations, it might have seemed natural to treat the approach flow in the square duct first, followed by that in the contraction and finally the flow in the rectangular duct downstream of the sudden expansion. In fact, in this paper, we do the reverse and thereby reflect the chronological sequence in which the experiments were carried out. As will become apparent, the flow downstream of the expansion was found to have a completely different character from that expected and this prompted a subsequent exploration of the flow within the contraction. We had anticipated the latter would not differ qualitatively from the flow such a contraction would produce for a Newtonian fluid with a 'top-hat' profile at outlet (i.e. the sudden-expansion inlet). As will be seen, the contraction flow has features so extreme and never previously reported that we felt the need, as a final step, to confirm that the approach flow itself was unremarkable. Prior to presenting the flow measurements, we present and discuss measurements of the rheology of the working liquid, a $0.05 \%$ by weight aqueous solution of a PAA.

\section{Experimental rig and instrumentation}

The flow loop used for the present experiments is identical to that used by Escudier et al. (2002), which is a modified version of that used by Escudier \& Smith (2001) for their square-duct investigation. The flow loop is shown schematically in figure 1. Flow was provided by a progressive cavity pump [1] (Mono type E101, maximum flow rate $0.025 \mathrm{~m}^{3} \mathrm{~s}^{-1}$; numbers in brackets refer to the components shown in figure 1) fed directly from a stainless steel tank of 


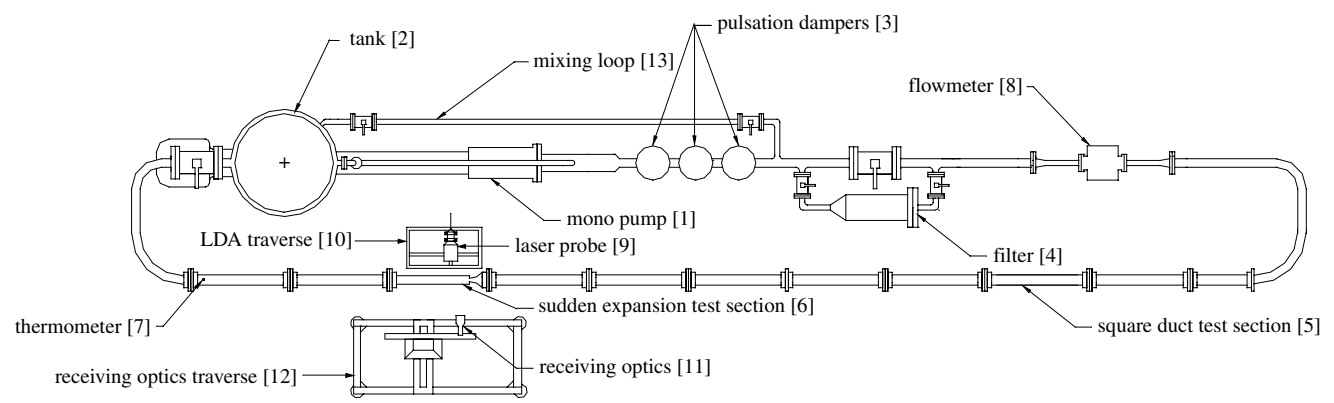

Figure 1. Schematic of flow loop.

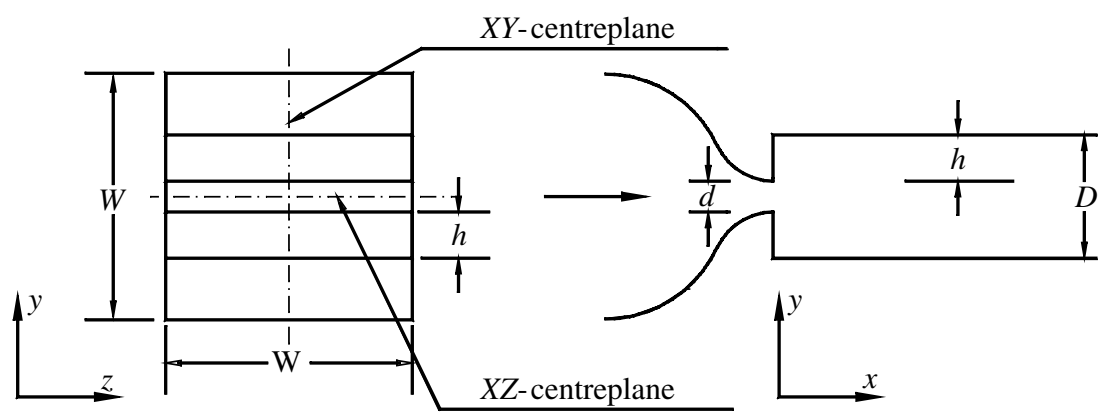

Figure 2. Geometry of plane sudden expansion.

$0.5 \mathrm{~m}^{3}$ capacity [2]. Three pulsation dampers [3] located immediately downstream of the pump outlet were provided to smooth out the flow and remove any pulsations prior to entry into the square duct test section [5]. Prior to entry to the test section, a honeycomb section was included to straighten the flow. The square duct consisted of 10 modules each of length $1.2 \mathrm{~m}$ and with a square internal cross-section of side length $w=80 \mathrm{~mm}$. Each module was constructed from stainless steel plate, machined and ground to size and then assembled with screws and a bonding agent. The expansion module [6] (shown schematically in figure 2) replaced module nine of the square duct arrangement and so was located $9.6 \mathrm{~m}$ (120 hydraulic diameters) from the inlet connection. The expansion was preceded by a short (53.5 mm length), gradual, plane contraction (40 mm concave radius followed by $20 \mathrm{~mm}$ convex radius). The duct width $w$ throughout, including the contraction, was $80 \mathrm{~mm}$, the inlet height at exit from the contraction $d$ was $10 \mathrm{~mm}$ and the step height $h$ was $15 \mathrm{~mm}$. The height $D$ of the downstream rectangular duct following the expansion was $40 \mathrm{~mm}$. The sidewalls of the contraction, the downstream duct and the square duct just upstream of the contraction were all made of borosilicate glass to permit velocity measurements using a laser Doppler anemometer (LDA) [9]. Distributions of streamwise velocity $(U)$ were obtained within the $X Y$-centre plane from vertical traverses, i.e. in the $y$-direction, at nine streamwise locations within the downstream duct corresponding to $x / h$ values of $0,1,2,3,4,5,6,8$ and 12 . For convenience, throughout the text we refer to these velocity distributions as vertical profiles. An additional vertical profile was measured just within the contraction at $x / h=-3$. Distributions of streamwise velocity were also obtained 
from horizontal traverses across the duct, i.e. in the $z$-direction along, $y / w=0$, within the $X Z$-centre plane, hereafter referred to as horizontal profiles, both within the contraction $(x / h=-3,-1.67,-1)$ and downstream of the expansion $(x / h=0,1,2,3,4,5,6,8$ and 12$)$. The approach flow within the square duct was mapped at a streamwise location approximately $240 \mathrm{~mm}$ (i.e. three hydraulic diameters) upstream of the gradual contraction by measuring distributions of streamwise velocity along horizontal traverses at $y / w=0,0.125,0.25$ and 0.375 and along a vertical traverse at $z / w=0$.

A Dantec Fibreflow LDA system was used for the velocity measurements and comprised a Dantec 60X10 probe and a Dantec 55X12 beam expander in conjunction with a Dantec Burst Spectrum Analyser signal processor (model $57 \mathrm{~N} 10$ ). The beam separation at the front lens was $51.5 \mathrm{~mm}$ and the lens focal length $160 \mathrm{~mm}$ which produced a measurement volume in water with principal axis of length $0.21 \mathrm{~mm}$ and diameter $0.02 \mathrm{~mm}$. Ensemble averages at each measurement location were formed from not less than 9500 velocity samples. The total uncertainty in the mean velocity values is estimated to be in the range 3-4\% (e.g. Poole \& Escudier 2004).

A platinum-resistance thermometer [7] mounted in the final module of the test section was used to monitor the fluid temperature to an accuracy of $\pm 0.1^{\circ} \mathrm{C}$. The bulk flow rate $\dot{Q}_{\mathrm{F}}$ was measured using a Fischer and Porter electromagnetic flow meter [8] (model 10D1) incorporated in the flow loop upstream of the square duct with the flow-meter output signal recorded via an Amplicon PS 30AT A/D converter.

Steady shear and oscillatory shear rheological measurements were carried out using a TA Instruments Rheolyst AR 1000N controlled-stress rheometer at a temperature of $20{ }^{\circ} \mathrm{C}$ which was also the average temperature of the fluid within the flow loop for the duration of the experimental runs. The rheological characterization included measurements of shear viscosity $\mu(\dot{\gamma})$, the storage modulus $G^{\prime}$ and the loss modulus $G^{\prime \prime}$. Temperature control of the rheometer is achieved via a plate that uses the Peltier effect to control the temperature of the sample to within $\pm 0.1{ }^{\circ} \mathrm{C}$. The extensional properties of the polymer were investigated using a Capillary Break-up Extensional Rheometer (CaBER) supplied by Thermo Haake. The diameter of the end platens is $D_{0}=4 \mathrm{~mm}$, the initial aspect ratio $\left(\Lambda=h_{0} / D_{0}\right) 0.5$ and the final axial separation of the plates was $8.36 \mathrm{~mm}$. The midpoint diameter is measured using a laser micrometer with a resolution of approximately $50 \mu \mathrm{m}$.

\section{Working fluid characteristics}

The working fluid used in this investigation was a $0.05 \%$ by weight aqueous solution of a PAA, Separan AP273 E supplied by Floerger. The solvent was filtered tap water with 100 ppm of $40 \%$ formaldehyde solution added to retard bacterial degradation. Approximately $0.25 \mathrm{~g}$ of Timiron seeding particles were added to the working fluid, total volume $575 \mathrm{l}$, to improve the LDA signal quality. PAA was chosen as it is highly viscoelastic, is optically transparent, thereby permitting LDA measurements, and has been used extensively in previous investigations in our laboratory (e.g. Escudier et al. 1999; Poole \& Escudier 2004). This polymer is generally regarded (Walters et al. 1990) as being 


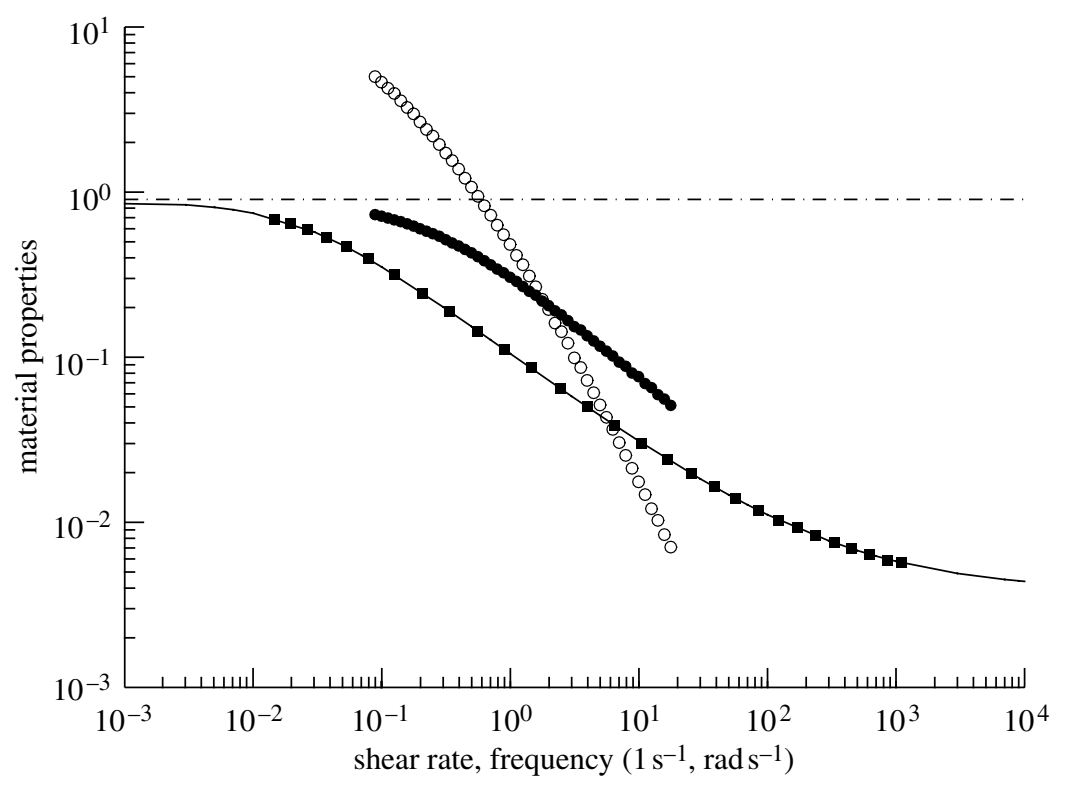

Figure 3. Rheological material functions of the $0.05 \%$ PAA solution: filled square, shear viscosity $\mu_{\mathrm{S}}(\mathrm{Pa} \mathrm{s})$; filled circle, dynamic viscosity $\eta^{\prime}\left(=G^{\prime \prime} / \omega\right)(\mathrm{Pa} s)$; open circle, dynamic rigidity $\left(=2 G^{\prime} / \omega^{2}\right)$ $\left(\mathrm{Pa} \mathrm{s}^{2}\right)$; solid line, Carreau-Yasuda model; dash-dotted line, $\Sigma n_{i}=0.903 \mathrm{~Pa}$ s.

'very flexible' in its molecular structure and it is this flexibility which gives the liquid more pronounced elastic properties than other water-soluble polymers such as xanthan gum and carboxymethylcellulose. The average molecular weight for the PAA used in this study, ascertained using gel phase chromatography, was $1.94 \times 10^{6} \mathrm{~g} \mathrm{~mol}^{-1}$ with a polydispersity of 1.05 .

The measured shear-viscosity versus shear-rate data for PAA are shown in figure 3 together wth the Carreau-Yasuda model fit

$$
\mu_{\mathrm{CY}}=\mu_{\infty}+\frac{\mu_{0}-\mu_{\infty}}{\left(1+\left(\lambda_{\mathrm{CY}} \dot{\gamma}\right)^{a}\right)^{n / a}},
$$

where $\mu_{0}=0.880 \mathrm{Pas}$ is the zero-shear-rate viscosity, $\mu_{\infty}=0.00388 \mathrm{Pas}$ the infinite-shear-rate viscosity, $\lambda_{\mathrm{CY}}=42.4 \mathrm{~s}$ a constant which represents the onset of shear thinning, $n=0.572$ a power-law index and $a=1.11$ a parameter introduced by Yasuda et al. (1981). The parameters were determined using the leastsquares-fitting procedure outlined by Escudier et al. (2001).

At a PAA concentration of $0.05 \%$ the first-normal-stress difference $\left(N_{1}\right)$ values were below the sensitivity of the rheometer even at the highest shear rates. The results of measurements of the viscoelastic properties of the $0.05 \%$ PAA solution in oscillatory shear can be seen in figure 3 in terms of the dynamic rigidity $\left(=2 G^{\prime} / \omega^{2}\right)$ and the dynamic viscosity $\left(=G^{\prime \prime} / \omega\right)$. A linearity check was conducted to determine the linear viscoelastic region prior to the frequency sweep. The frequency sweep was performed at a shear stress of $0.02 \mathrm{~Pa}$, a value well within the linear regime and comparison with data at a higher shear stress, again within the linear regime, confirmed that the viscoelastic properties observed were independent of the shear stress. Since $G^{\prime}$ has to be zero for an inelastic liquid, it is evident that the oscillation data reveal a high degree of 
elasticity for the PAA solution. To obtain an estimate for the relaxation time for the solution we fitted a four-mode Maxwell model to the $G^{\prime}$ and $G^{\prime \prime}$ data (following Bird et al. 1987)

$$
G^{\prime}=\sum_{i}^{4} \frac{\eta_{i} \lambda_{i} \omega^{2}}{1+\left(\lambda_{i} \omega\right)^{2}}, \quad G^{\prime \prime}=\sum_{i}^{4} \frac{\eta_{i} \omega}{1+\left(\lambda_{i} \omega\right)^{2}},
$$

which yields relaxation times: $\lambda_{i}=8.1,1.4,0.23$ and $0.02 \mathrm{~s}$ and viscosity constants $\eta_{i}=0.379,0.339,0.133$ and $0.0520 \mathrm{~Pa}$. The sum of these viscosity constants is shown in figure 3 as a dashed line and is equal (within $3 \%$ ) of the $\mu_{0}$ value determined from the Carreua-Yasuda fit. In $\S 4$, we shall use these values to estimate an average relaxation time $\lambda$ for the fluid in order to define a Deborah number for the flow.

In order to investigate the extensional properties of our PAA solution we employed the capillary break up technique using a CaBER supplied by Thermo Haake. In this technique, pioneered by Bazilevsky et al. (1990), a cylindrical liquid bridge of the 'test' fluid is formed between two parallel circular platens $4 \mathrm{~mm}$ in diameter. An axial step strain is then applied (i.e. the end plates are rapidly pulled apart to a fixed separation) which results in the formation of an elongated liquid thread. The thread diameter reduces due to surface tension $(\sigma)$ and information about the extensional properties of the liquid can be deduced from the evolution of the filament midpoint diameter $\left(D_{\text {mid }}(t)\right)$ which is monitored using a laser micrometer. Following the ideas proposed by Schümmer \& Tebel (1983), it is possible to perform a simple force balance on the fluid element and then obtain an 'apparent' extensional viscosity $\left(\eta_{\text {app }}\right)$ according to the following equation (Anna \& McKinley 2001)

$$
\eta_{\text {app }}=\frac{2 \sigma / D_{\text {mid }}(t)}{\dot{\varepsilon}(t)}=\frac{-\sigma}{\mathrm{d} D_{\text {mid }} / \mathrm{d} t} .
$$

However, this approach leads to an estimate of a transient extensional viscosity and we prefer to follow the route advocated by McKinley and co-workers (Anna \& McKinley 2001; Rodd et al. 2005) to estimate a characteristic extensional relaxation time from the data for the evolution of the filament diameter. For our polymer solution this data is shown in figure 4 . For 'intermediate elastic times', i.e. after viscous effects have become negligible and before finite extensibility of the polymer dumbbells becomes important (Anna \& McKinley 2001), the filament diameter for a polymer solution should decay exponentially in time

$$
D_{\text {mid }}(t)=\left(\frac{G D_{1}^{4}}{2 \sigma}\right)^{1 / 3} \exp \left[\frac{-t}{3 \lambda_{\mathrm{EX}}}\right],
$$

where $G$ is the elastic modulus, $D_{1}$ is the midpoint diameter following cessation of stretching and $\lambda_{\mathrm{EX}}$ is a characteristic relaxation time. This relationship has been utilized by a number of workers to determine the relaxation time for many different polymer solutions over a range of concentrations and molecular weights (Bazilevsky et al. 1990; Stelter et al. 2000; Anna \& McKinley 2001; Rodd et al. 2005). We have fitted equation (3.4) to the CaBER data for our $0.05 \%$ PAA solution to the 'intermediate' times (in this case in the range $0.3 \mathrm{~s}<t<1.5 \mathrm{~s}$ ) over which it is valid and this fit, which yields a relaxation time $\lambda_{\mathrm{EX}}=0.34 \mathrm{~s}$, is included as a solid line in figure 4 . 


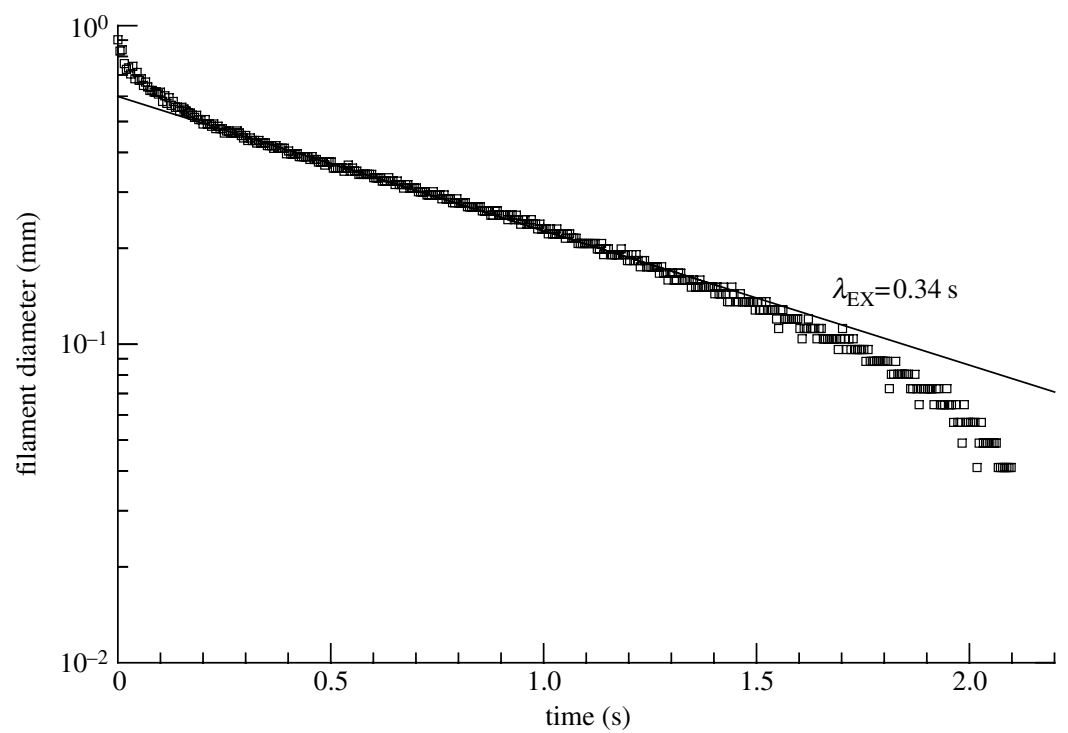

Figure 4. Exponential decay of fluid filament diameter for 0.05\% PAA (CaBER experiments).

\section{Estimation of Reynolds and Deborah numbers}

To define a Reynolds number for the PAA flow, we adopt the bulk velocity $U_{\mathrm{B}}$ determined from the volumetric flow rate $\left(U_{\mathrm{B}}=\dot{Q}_{\mathrm{F}} / w d=0.27 \mathrm{~m} \mathrm{~s}^{-1}\right)$ while for the length scale we can select either the expansion-inlet height $(d=0.01 \mathrm{~m})$ or the step height $(h=0.015 \mathrm{~m})$. The density of the polymer solution was essentially that of the solvent, water. For a shear-thinning liquid no single viscosity can be identified which completely characterizes the fluid and so any definition of a single Reynolds number to characterize the flow is somewhat arbitrary. However, several well-defined 'characteristic' shear rates $\dot{\gamma}_{\mathrm{CH}}$ can be defined from each of which a corresponding characteristic viscosity $\mu_{\mathrm{CH}}$ can be determined (using in this case the Carreau-Yasuda model). The various methods of estimating Re are shown in table 2 .

The difficulties in selecting an appropriate Reynolds number for a strongly shear-thinning liquid are highlighted by table 2 , where it can be seen that the Reynolds number ranges between about 3 and 1026 (i.e. almost three orders of magnitude) depending upon the choice of $\dot{\gamma}_{\mathrm{CH}}$. The minimum and maximum possible values (for given velocity and length scales) of the Reynolds number are based upon the shear rate tending to either zero or infinity and are best used as a guide. The shear rate at inlet $\left.\dot{\gamma}_{\mathrm{CH}}\right|_{\text {inlet }}$ has a precise physical interpretation but requires a priori detailed knowledge of the flow field to determine its value and cannot easily be used to compare to numerical simulations because, unless the actual and calculated velocity profiles at inlet agree, the Reynolds numbers will differ. In view of the foregoing we favour the use of a more simplistic Reynolds number $\operatorname{Re}_{d}$ based upon a viscosity corresponding to a characteristic shear rate defined from bulk-flow quantities, i.e. $\dot{\gamma}_{\mathrm{CH}}=U_{\mathrm{B}} / h$.

The Deborah number $D e$ is a ratio of two characteristic times, one representing the relaxation processes occurring in the flow of a viscoelastic 
Table 2. Reynolds-number estimates for various characteristic shear rates

\begin{tabular}{lllc}
\hline$\dot{\gamma}_{\mathrm{CH}}\left(\mathrm{s}^{-1}\right)$ & $\mu_{\mathrm{CH}}(\mathrm{Pas})$ & $\operatorname{Re}_{d}=\rho U_{\mathrm{B}} d / \mu_{\mathrm{CH}}$ & $\operatorname{Re}_{h}=\rho U_{\mathrm{B}} h / \mu_{\mathrm{CH}}$ \\
\hline$\rightarrow 0$ & 0.880 & 3.1 & 4.5 \\
$\dot{\gamma}_{\mathrm{CH}}=U_{\mathrm{B}} / h \approx 18$ & 0.0233 & 115 & 172 \\
$\dot{\gamma}_{\mathrm{CH}}=U_{\mathrm{B}} / d \approx 27$ & 0.0192 & 138 & 209 \\
$\left.\dot{\gamma}_{\mathrm{CH}}\right|_{\text {Inlet }}=\left.(\mathrm{d} U / \mathrm{d} y)\right|_{\text {Wall }} \approx 400$ & 0.0071 & 376 & 564 \\
$\rightarrow \infty$ & 0.0039 & 684 & 1026 \\
\hline
\end{tabular}

liquid, which we here take as the average relaxation time $\lambda(=3.9 \mathrm{~s})$ from the Maxwell model fit to the $G^{\prime}, G^{\prime \prime}$ data presented in $\S 3$ and estimated using the expression

$$
\lambda=\frac{\sum_{i}^{4} \lambda_{i} \eta_{i}}{\sum_{i}^{4} \eta_{i}}
$$

and a characteristic time for the flow itself, which we take as $h / U_{\mathrm{B}}$. We find, therefore, $D e=\lambda U_{\mathrm{B}} / h=69$. An 'extensional' relaxation time, determined from the CaBER measurements, $\lambda_{\mathrm{EX}}=0.34 \mathrm{~s}$, gives an alternative $D e=\lambda_{\mathrm{EX}} U_{\mathrm{B}} / h=7$. Both of these Deborah numbers are consistent with a flow in which viscoelastic effects will play a major role.

\section{Discussion}

(a) Flowfield downstream of the plane sudden expansion

The streamwise variation of the vertical velocity profiles downstream of the expansion inlet are plotted in figure $5 a, b$. Also shown are the loci of zero velocity to provide a visual guide to the lengths of the top and bottom recirculation regions and also to demonstrate the essentially symmetric nature (at least upstream of reattachment) of the flow field in the vertical symmetry plane of the geometry. Streamlines would, of course, show this much more satisfactorily but cannot be constructed due to the three-dimensionality of the flow field. The apparent flow rate $\dot{Q}_{\mathrm{A}}$ determined at each streamwise location by numerical integration of the velocity profiles assuming two-dimensional flow, is given in table 3 and reveals clearly that the flow is far from two-dimensional: within 12 step heights the apparent flow rate has increased by a factor of 3.3. The progressive increase of the apparent flow rate with downstream distance, combined with an inspection of the velocity profiles (figure $5 a, b$ ), shows that liquid is being forced into the measurement plane (i.e. the $X Y$-centre plane) as the flow progresses downstream.

Expansion of the high-velocity core is evident immediately downstream of the expansion inlet $(0<x / h<1)$ as has been observed previously at much lower Reynolds numbers (Townsend \& Walters 1994), and must be related to the elastic energy stored in the polymer molecules being released after the contraction outlet. The reattachment lengths $x_{\mathrm{R}}$ for the top and bottom recirculation regions differ by less than $4 \%, 5.2$ step heights (top) compared to 5.4 step heights (bottom), and show that the asymmetry, although greatly 

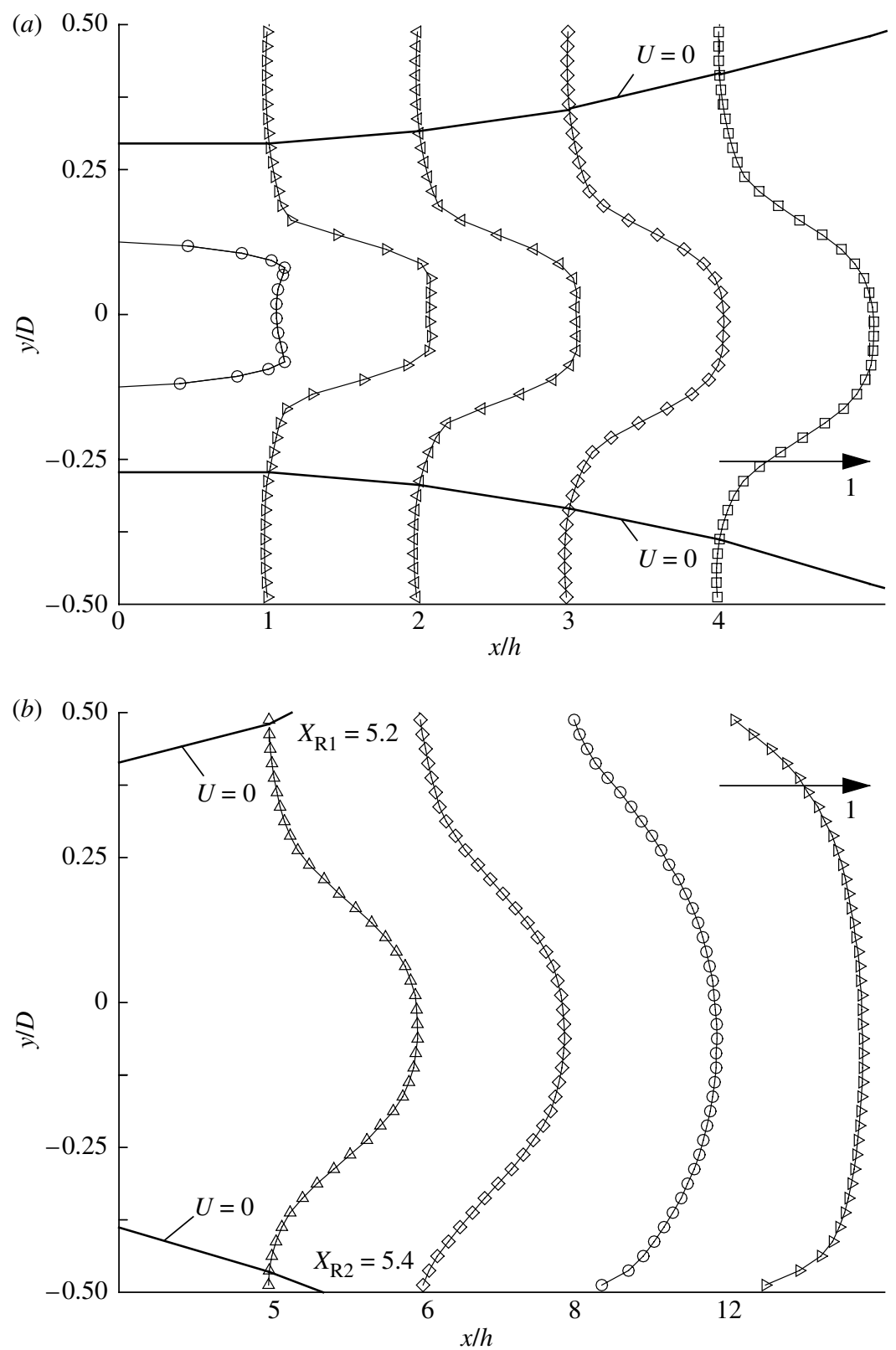

Figure 5. Vertical profiles of velocity downstream of expansion. (a) $0 \leq x / h \leq 4$, (b) $5 \leq x / h \leq 12$. Unit arrow shows scale for velocity, $U / U_{\mathrm{B}}$

reduced compared to a Newtonian fluid flow at the same Reynolds number (Drikakis 1997; determined the critical Reynolds number to be 53 for a Newtonian fluid flow through a 'two-dimensional' $1: 4$ plane sudden expansion), is not completely eliminated for this viscoelastic fluid flow. The asymmetry continues to develop with downstream distance as is clearly evident in the profiles at $x / h=8$ and 12 (figure $5 b$ ). 
Table 3. Apparent flow rates at various streamwise locations in the XY-centre plane $\left(\dot{Q}_{\mathrm{F}}=0.778 \mathrm{~m}^{3} \mathrm{~h}^{-1}\right)$

\begin{tabular}{llllllllll}
\hline \multicolumn{2}{c}{$x / h=0$} & 1 & 2 & 3 & 4 & 5 & 6 & 8 & 12 \\
\hline$\dot{Q}_{\mathrm{A}}\left(\mathrm{m}^{3} \mathrm{~h}^{-1}\right)$ & 0.718 & 0.817 & 0.931 & 1.050 & 1.233 & 1.418 & 1.631 & 2.087 & 2.358 \\
$\dot{Q}_{\mathrm{A}} / \dot{Q}_{\mathrm{F}}$ & 0.923 & 1.05 & 1.20 & 1.35 & 1.58 & 1.82 & 2.10 & 2.68 & 3.03 \\
\hline
\end{tabular}

To investigate further the three-dimensionality of the flowfield downstream of the sudden expansion, and the mechanism responsible for the increase in apparent flow rate along the $X Y$-centre plane with downstream distance, horizontal velocity profiles were taken at discrete streamwise locations $(x / h=0$, $1,2,3,4,5,6,8$ and 12) and can be seen in figure $6 a, b$. These profiles show that the increase with downstream distance in apparent flow rate observed in the $X Y$ centre plane (table 3 ) is a consequence of fluid being 'fed' from the flow near the sidewalls. Within figure $6 a, b$ (and all subsequent figures) filled symbols represent data points reflected about the respective centre plane (i.e. $X Y$ or $X Z$ ) and, in this case, demonstrate that there is no discernible asymmetry of the flow about the $X Y$-centre plane. What is remarkable is that by $x / h=12$ the majority of the flow is contained within a narrow vertical jet of fluid centred around the $X Y$ centre plane with almost stagnant fluid on either side.

\section{(b) Flow field within the gradual contraction}

In addition to the complicated three-dimensional structure, which develops progressively downstream of the expansion, both the vertical profile of velocity at inlet (i.e. $x / h=0$ figure $5 a$ ) and, to an even greater extent, the corresponding horizontal profile (figure 6) exhibit unexpected velocity overshoots. To identify the origin of this behaviour we investigated the flow field within the gradual contraction itself. Figure 7 shows horizontal profiles of velocity throughout the contraction (at $x / h=-3,-1.67,-1$ and 0 ) and also vertical profiles near the entrance $(x / h=-3)$ and at the exit $(x / h=0)$ of the contraction. As in figure 6 , to provide a visual guide to the side-to-side and top-to-bottom flow symmetry within the contraction, mirror-image points (about either $z / w=0$ or $y / w=0$ ) are included as filled symbols. Due to the shape of the contraction, optical access for the laser beams of the LDA system is restricted within the contraction near the top and bottom curved walls, which is why no vertical profiles were obtained for intermediate streamwise locations. Note that the curtailed appearance of the vertical profile just inside the contraction (i.e. at $x / h=-3$ ) is a consequence of the reduced contraction height at this location.

As a basis for comparison, numerically simulated profile shapes for a Newtonian fluid and for a shear-thinning fluid with viscosity based upon the Carreau-Yasuda model fit to the $0.05 \%$ PAA data of figure 3 are shown in figure $7 b$. These profiles were obtained for $\operatorname{Re}_{h}=200$ using the fully three-dimensional, finite-volume code previously reported by Issa \& Oliveira (1994) in which secondorder discretization methods are used throughout, except for the important convective terms in the equations of motion where a third-order high-resolution scheme is applied (Alves et al. 2003). The simulation for the Newtonian fluid 

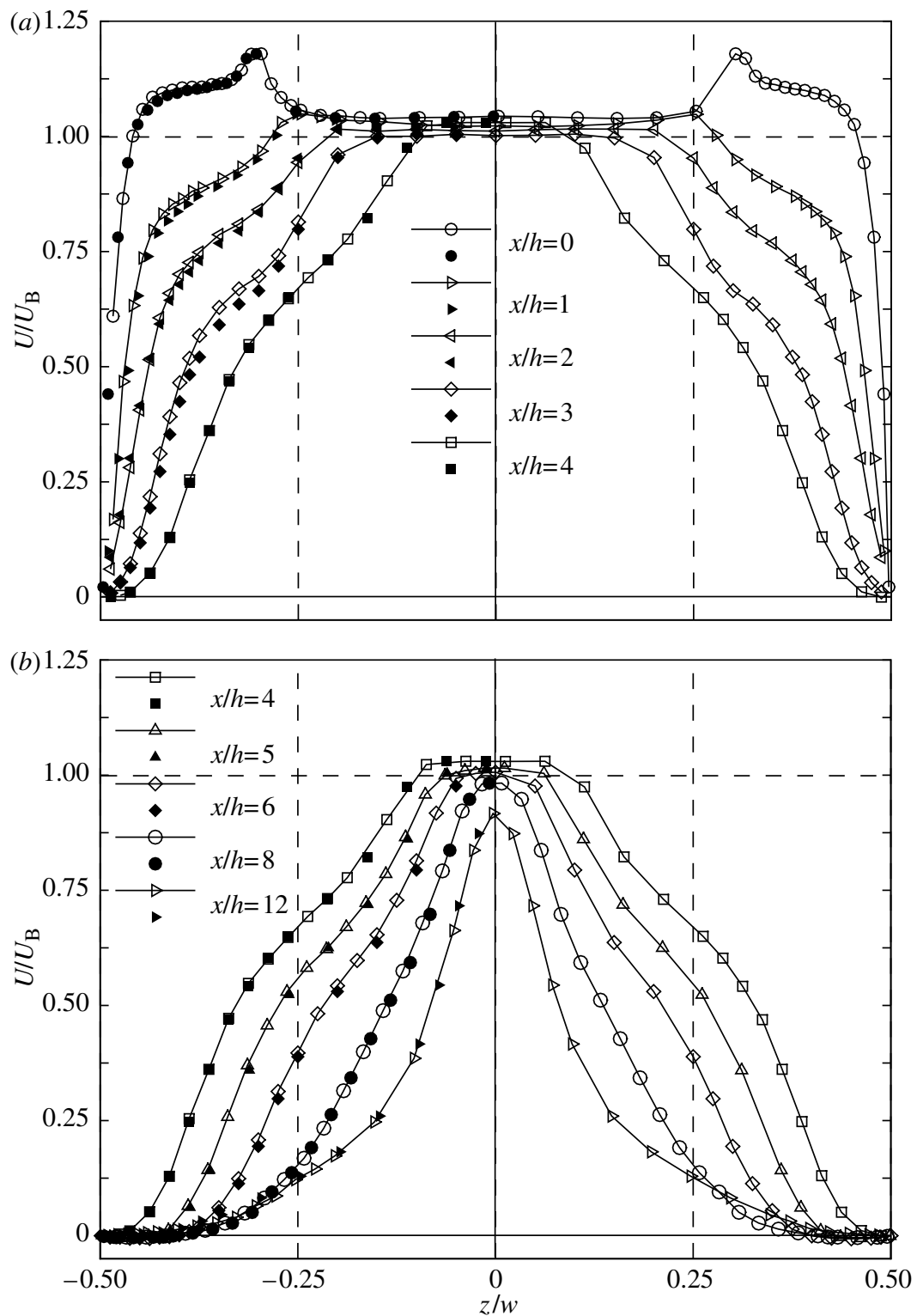

Figure 6. Horizontal profiles of velocity downstream of expansion. (In this and all subsequent figures filled symbols represent reflected data points about respective centre plane.) (a) $0 \leq x / h \leq 4$, (b) $4 \leq x / h \leq 12$.

shows the expected 'parabolic' shape of the profiles just within the contraction, in excellent agreement with the theoretical solution for fully developed flow in a square duct (Shah \& London 1978). At exit the horizontal profile is symmetrical and has flattened while the asymmetry just evident in the vertical profile is the result of the top-to-bottom asymmetry of the flow downstream of the expansion. For the Carreau-Yasuda fluid, the horizontal profile is even flatter but in all respects only slightly different to that for the Newtonian fluid. 

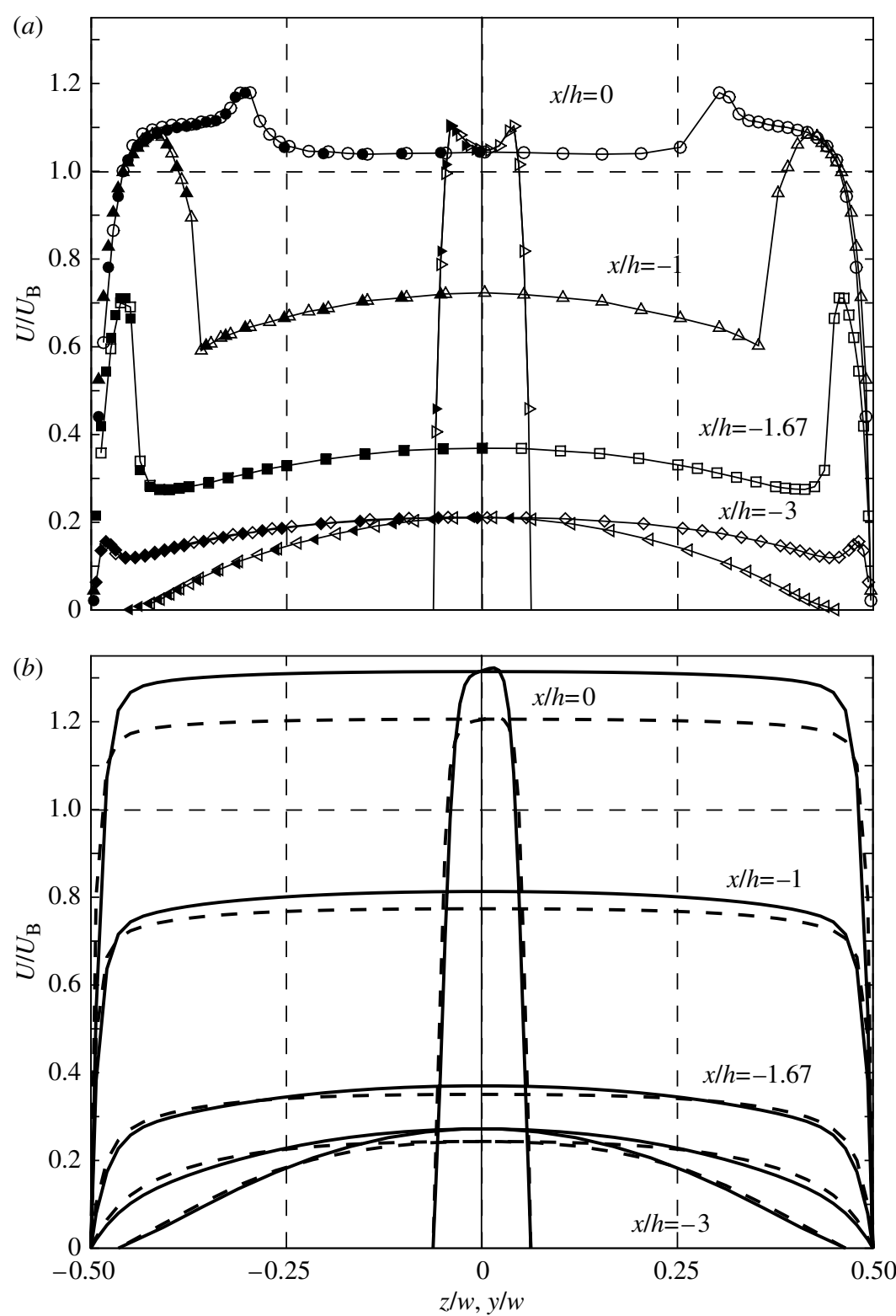

Figure 7. Horizontal (open circle, open triangle, open square, open diamond) and vertical (right open triangle, left open triangle) profiles of velocity through the gradual contraction. (a) Experimental results for $0.05 \%$ PAA, (b) numerical simulations for Newtonian (full line) and Carreau-Yasuda model (broken line) fluid flow at $\operatorname{Re}_{h}=200$.

In contrast to the numerically simulated flow of the inelastic fluids, the measured vertical profile for the PAA solution at $x / h=0$ displays a velocity overshoot $\left(1.1 U_{\mathrm{B}}\right)$ near the wall and a slight dip in the velocity on the centreline (i.e. the $X Y$-centre plane at $y / w=0)$ to $1.05 U_{\mathrm{B}}$. The horizontal profiles between the parallel walls of the contraction show that within the central section of the duct $(-0.25<z / w<0.25)$ the flow is consistent with what would be expected for 
the flow of a Newtonian fluid flow: as the area decreases, the velocity increases, and at outlet the velocity in this section of the duct is practically uniform and two-dimensional. Nearer to the flat sidewalls, the shape of the velocity profiles is remarkably different and unexpected. At the furthest upstream location $(x / h=-3)$ a small velocity 'overshoot' occurs near the walls. As the flow progresses downstream, this 'overshoot' grows in both magnitude (at $x / h=-1.67$ the peak value is almost double that of the velocity on the centreline) and width. The horizontal profile at $x / h=-1$ in figure 7 is the most striking. The transition between the central 'two-dimensional' part of the flow and the overshoot near the wall involves an abrupt change in gradient from negative and low in the central region to positive and very steep as the peak is approached, $c a \approx 100 \mathrm{~s}^{-1}$, which is about the same order of magnitude as the maximum shear rate, where the shear layer detaches from the step into the expansion figure $5 a$. The mechanism which produces the 'cat's ears' shape of these profiles, which so far as we are aware have not been observed hitherto, is unclear but is almost certainly related to the first and second normal-stress differences produced which increase with increasing shear rate. It is, of course, significant that there is no evidence of velocity overshoots in the Carreau-Yasuda simulation so that it can be concluded that these cannot be a consequence of the shear thinning alone. With viscoelastic fluids the possibility of elastic instabilities due to curved streamlines cannot a priori be ruled out as the triggering mechanism for the unusual flow patterns reported here. It is well known that when the Reynolds number is sufficiently high, a boundary layer developing in a Newtonian fluid over a concave surface gives rise to Görtler vortices, a pattern of paired streamwise counter-rotating vortices arranged along the main flow direction. Similar vortex patterns have been observed with viscoelastic fluid flow, for example in flows through contractions as documented by Chiba and co-workers in a series of papers which include Chiba et al. $(1992,1995)$ and Chiba \& Nakamura (1997). In such cases the instability is generated by the competing effects of elastic tension along a streamline coupled with streamline curvature. Chiba et al.'s results are very clear in showing that Görtler-type vortices are formed only after the onset of a 'diverging' streamline pattern which greatly increases the curvature of the streamlines upstream of the contractions. In our case the streamline curvature is determined solely by the curvature of the contraction wall, which since $(w / 2) / R_{i}$ (i.e. duct half width/radius of curvature of concave section of gradual contraction) is equal to one, the ratio of the 'effective' boundary layer thickness to the radius of curvature is likely to be much less than one. As a consequence, Görtler vortices are neither expected nor observed. The quantitative criterion for elastic instabilities developed by McKinley et al. (1996) is also useful here. Basically it predicts an instability when $\sqrt{D e \cdot W e}>8$, for a geometry having similarities with the present one. The Deborah number $D e$ is defined in terms of the streamline curvature, that is $D e=\lambda U_{\mathrm{SD}} / R_{i}$, and the Weissenberg number $W e$ with a characteristic shear rate, $W e=\lambda \dot{\gamma}=\lambda U_{\mathrm{SD}} /(w / 2)$. In our case $R_{i}=40 \mathrm{~mm}, w=80 \mathrm{~mm}$ and so both $W e$ and $D e$ take the same value. With $\lambda=3.9 \mathrm{~s}$ (i.e. our longest estimate of the fluid relaxation time) or $\lambda_{\mathrm{EX}}=0.34 \mathrm{~s}$ (i.e. the relaxation time determined from extensional measurements), and an average velocity $U_{\mathrm{SD}}=U_{\mathrm{B}} /(w / d)=0.27 / 8=0.034 \mathrm{~m} \mathrm{~s}^{-1}$, we obtain $\sqrt{D e \cdot W e}=$ 3.3 or 0.29 (depending on the choice of $\lambda$ or $\lambda_{\mathrm{Ex}}$ for the relaxation time), i.e. significantly lower than the critical value of 8 for the onset of Görtler-type vortex 
formation. This instability can, therefore, be discounted as the triggering mechanism for the 'cat's ears'. This outcome is not surprising because what we observe is a global flow feature occupying the whole half-section of the duct, whereas the Görtler-type vortices observed by Chiba et al. consisted of a number of small repeating flow structures (vortex tubes) having a characteristic wavelength much smaller than the duct spanwise dimension.

What remains unclear is why the overshoot appears to be far stronger along the flat sidewalls compared with the top and bottom curved walls of the contraction. Although our experimental arrangement did not allow us to make vertical traverses within the contraction, it is clear that the vertical velocity profile at entry $(x / h=-3)$ shows no sign of an overshoot, and the profile at exit $(x=0)$ shows only a slight overshoot immediately adjacent to the top and bottom surfaces of the contraction exit.

\section{(c) Approach-flow within the square duct}

Since, it might be thought that the cat's ears velocity profiles are not in fact produced within the contraction but are already present within the upstream approach flow within the square duct and only amplified by the contraction, the flow field within the square duct was mapped out three hydraulic diameters upstream of the gradual contraction. The results are presented in figure 8 . The velocity profiles (in this figure normalized by the bulk velocity within the square duct, i.e. $\left.U_{\mathrm{SD}}=\dot{Q}_{\mathrm{F}} / W^{2}\right)$ are similar to theoretical profiles calculated with a power-law model and are entirely consistent with fully developed laminar flow of a shear-thinning viscoelastic liquid (Wheeler \& Whissler 1966; Bird et al. 1987) and symmetric about both the $X Y$ and $X Z$-centre planes. We also note that the velocity profiles obtained from the Carreau-Yasuda simulation within the square duct (essentially the profiles at $x / h=-3$ shown in figure $7 b$ ), although flatter than the equivalent Newtonian profiles as might be expected from purely shearthinning considerations, are not identical to the experimental results. This difference suggests that, even at a relatively low-polymer concentration (0.05\%), this fluid produces second normal-stress differences large enough to induce secondary motion within the square duct, which in turn alter the streamwise velocity structure. This secondary motion in fully developed laminar flow of a viscoelastic fluid through a square duct was first investigated numerically by Wheeler \& Whissler (1966). More recently, Gervang \& Larsen (1991) and Debbaut et al. (1997) showed that the magnitude of the secondary-flow velocities are typically two orders of magnitude lower than that of the streamwise velocity. In the current experiments, we estimate that the maximum secondary flow velocities would be of order $10 \mu \mathrm{m} \mathrm{s}^{-1}$, below the resolution of our LDA system. However, as there is no evidence of velocity overshoots near the sidewalls, we conclude that the cat's ears are a consequence of viscoelastic and shear-thinning influences on the fluid as it flows through the contraction.

\section{Conclusions}

The results have been reported of an experimental investigation of the laminar flow $\left(R e_{d} \approx 115\right.$ ) of a viscoelastic liquid ( $D e=7$ or 69 , dependent on choice of relaxation time), a $0.05 \%$ aqueous solution of a PAA, Separan AP 273 E, through 


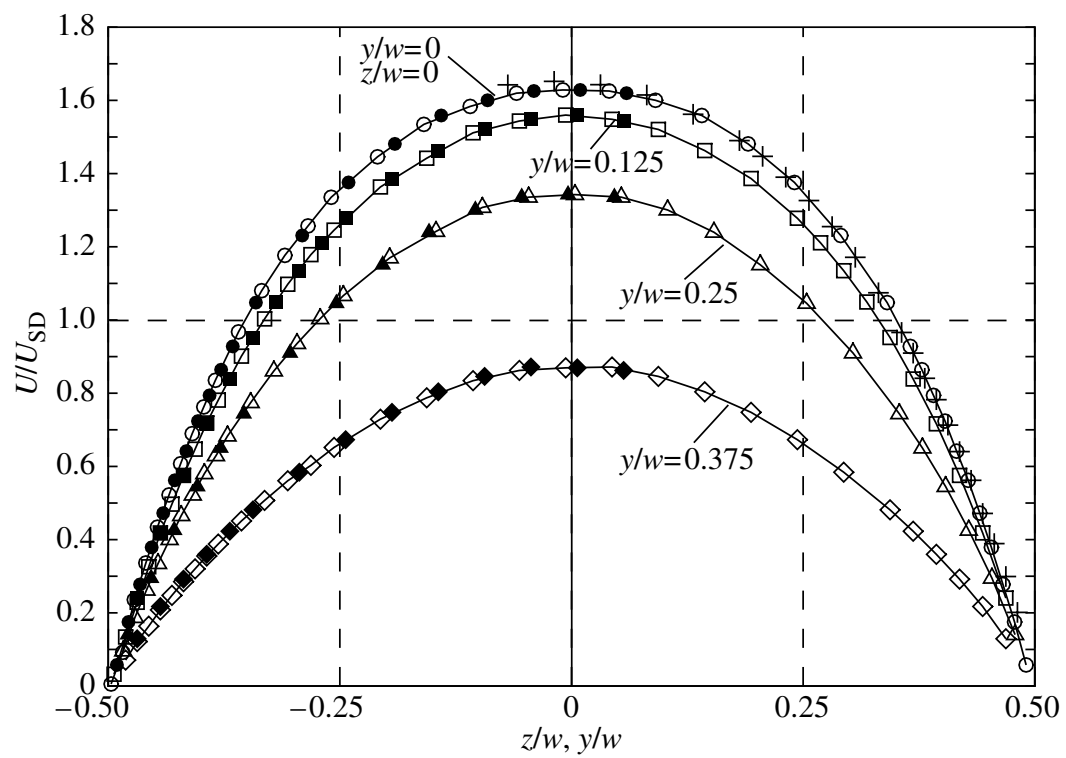

Figure 8. Profiles of velocity along horizontal lines at various heights (open circle, open square, open triangle, open diamonds) and a vertical profile of velocity $(+)$ at the downstream end of the square duct (normalized by bulk velocity within square duct $U_{\mathrm{SD}}$ ).

a plane sudden expansion, of expansion ratio $R=D / d=4$. The expansion duct is preceded by a gradual contraction, with an outlet of aspect ratio $8: 1$, upstream of which is a long (120 hydraulic diameters) square duct.

Downstream of the sudden expansion a complex three-dimensional flow is observed. The top-to-bottom asymmetry seen in the flow of a Newtonian fluid at a comparable Reynolds number is significantly reduced although a slight difference persists in the lengths of the two recirculation-regions and, more significantly, the vertical distributions of velocity become increasingly asymmetric downstream of reattachment. Integration of the vertical velocity profiles reveals significant departures from two-dimensionality with the apparent flow rate along the $X Y$-centre plane increasing progressively with downstream distance from the expansion. The corresponding horizontal profiles in the $X Z$ centre plane show that this increase is the consequence of the development of a strongly peaked jet-like flow structure, symmetric about the $X Y$-centre plane, with low-velocity fluid on either side.

The fully developed flow in the square duct upstream of the gradual contraction is unremarkable but flow through the contraction that precedes the expansion is spectacularly different from that expected for a Newtonian fluid. As the flow progresses through the contraction, velocity overshoots develop adjacent to the flat sidewalls of the contraction that first increase then decrease in magnitude. There is an abrupt change from the nearly two-dimensional flow in the central region of the contraction with extremely high-velocity gradients evident in the velocity overshoots. We have coined the term 'cat's ears' for the shape of these velocity profiles which, so far as we are aware, have not been reported hitherto. Only slight overshoots are seen in the velocity profiles, which develop within the vertical centre plane. 
We associate the three-dimensionality of the flow, both within the contraction and downstream of the expansion, with the normal-stress differences produced as a consequence of the viscoelasticity of the liquid.

The authors would like to express their gratitude to one of the referees who, in addition to bringing to our attention the papers of Chiba et al. and the work of McKinley et al. on purely elastic instabilities, made a number of insightful comments which assisted us in the interpretation of our results.

\section{References}

Alves, M. A., Oliveira, P. J. \& Pinho, F. T. 2003 A convergent and universally bounded interpolation scheme for the treatment of advection. Int. J. Numer. Methods Fluids 41, 47. (doi:10.1002/fld.428.)

Anna, S. L. \& McKinley, G. H. 2001 Elasto-capillary thinning and break up of model elastic liquids. J. Rheol. 45, 115. (doi:10.1122/1.1332389.)

Baloch, A., Townsend, P. \& Webster, M. F. 1996 On vortex development in viscoelastic expansion and contraction flows. J. Non-Newt. Fluid Mech. 65, 133. (doi:10.1016/0377-0257(96)01470-X.)

Bazilevsky, A. V., Entov, V. M. \& Rozhkov, A. N. 1990 Liquid filament micro-rheometer and some of its applications. In Third European Rheology Conference (ed. D. R. Oliver), p. 41. London: Elsevier Applied Science.

Bird, R. B., Armstrong, R. C. \& Hassager, O. 1987 Dynamics of polymeric fluids Fluid mechanics, vol. 1, 2nd edn. New York: Wiley-Interscience.

Cherdron, W., Durst, F. \& Whitelaw, J. H. 1978 Asymmetric flows and instabilities in symmetric ducts with sudden expansions. J. Fluid Mech. 84, 13.

Chiba, K. \& Nakamura, K. 1997 Instabilities in a circular entry flow of dilute polymer solutions. J. Non-Newt. Fluid Mech. 73, 67. (doi:10.1016/S0377-0257(97)00036-0.)

Chiba, K., Tanaka, S. \& Nakamura, K. 1992 The structure of anomalous entry flow patterns through a planar contraction. J. Non-Newt. Fluid Mech. 42, 315. (doi:10.1016/03770257(92)87016-5.)

Chiba, K., Ishida, R. \& Nakamura, K. 1995 Mechanism for entry flow instability through a forward-facing step. J. Non-Newt. Fluid Mech. 57, 271. (doi:10.1016/0377-0257(94)01335-F.)

Darwish, M. S., Whiteman, J. R. \& Bevis, M. J. 1992 Numerical modelling of viscoelastic liquids using a finite-volume method. J. Non-Newt. Fluid Mech. 45, 311. (doi:10.1016/03770257(92)80066-7.)

Debbaut, B., Avalosse, T., Dooley, J. \& Hughes, K. 1997 On the development of secondary motions in straight channels induced by the second normal stress difference: experiments and simulations. J. Non-Newt. Fluid Mech. 69, 255. (doi:10.1016/S0377-0257(96)01543-1.)

Drikakis, D. 1997 Bifurcation phenomena in incompressible sudden expansion flows. Phys. Fluids 9, 76. (doi:10.1063/1.869174.)

Durst, F., Melling, A. \& Whitelaw, J. H. 1974 Low Reynolds number flow over a plane sudden expansion. J. Fluid Mech. 64, 111.

Escudier, M. P. \& Smith, S. E. 2001 Fully developed turbulent flow of Newtonian liquids through a square duct. Proc. R. Soc. A 457, 911. (doi:10.1098/rspa.2000.0698.)

Escudier, M. P., Presti, F. \& Smith, S. E. 1999 Drag reduction in the turbulent pipe flow of polymers. J. Non-Newt. Fluid Mech. 81, 197. (doi:10.1016/S0377-0257(98)00098-6.)

Escudier, M. P., Gouldson, I. W., Pereira, A. S., Pinho, F. T. \& Poole, R. J. 2001 On the reproducibility of the rheology of shear-thinning liquids. J. Non-Newt. Fluid Mech. 97, 99. (doi:10.1016/S0377-0257(00)00178-6.)

Escudier, M. P., Oliveira, P. J. \& Poole, R. J. 2002 Turbulent flow through a plane sudden expansion of modest aspect ratio. Phys. Fluids 14, 3641. (doi:10.1063/1.1504711.)

Fearn, R. M., Mullin, T. \& Cliffe, K. A. 1990 Non-linear flow phenomena in a symmetric sudden expansion. J. Fluid Mech. 1990, 595. 
Gervang, B. \& Larsen, P. S. 1991 Secondary flows in straight ducts of rectangular cross section. J. Non-Newt. Fluid Mech. 39, 217. (doi:10.1016/0377-0257(91)80016-D.)

Halmos, A. L. \& Boger, D. V. 1976 Flow of viscoelastic polymer solutions through an abrupt 2-to-1 expansion. Trans. Soc. Rheol. 20, 253. (doi:10.1122/1.549413.)

Halmos, A. L., Boger, D. V. \& Cabelli, A. 1975 a The behavior of a power-law fluid flowing through a sudden expansion Part I. Numerical solution. AIChE J. 21, 540. (doi:10.1002/aic.690210316.)

Halmos, A. L., Boger, D. V. \& Cabelli, A. $1975 b$ The behavior of a power-law fluid flowing through a sudden expansion Part II. Experimental verification. AIChE J. 21, 550. (doi:10.1002/aic. 690210317.)

Issa, R. I. \& Oliveira, P. J. 1994 Numerical prediction of phase separation in two-phase flow through T-junctions. Comput. Fluids 23, 347. (doi:10.1016/0045-7930(94)90045-0.)

Manica, R. \& De Bortoli, A. L. 2004 Simulation of sudden expansion flows for power-law fluids. J. Non-Newt. Fluid Mech. 121, 35. (doi:10.1016/j.jnnfm.2004.03.009.)

McKinley, G. H., Pakdel, P. \& Oztekin, A. 1996 Rheological and geometric scaling of purely elastic flow instabilities. J. Non-Newt. Fluid Mech. 67, 19. (doi:10.1016/S0377-0257(96)01453-X.)

Missirlis, K. A., Assimacopoulos, D. \& Mitsoulis, E. 1998 A finite volume approach in the simulation of viscoelastic expansion flows. J. Non-Newt. Fluid Mech. 78, 91. (doi:10.1016/ S0377-0257(98)00057-3.)

Neofytou, P. \& Drikakis, D. 2003 Non-Newtonian flow instability in a channel with a sudden expansion. J. Non-Newt. Fluid Mech. 111, 127. (doi:10.1016/S0377-0257(03)00041-7.)

Oliveira, P. J. 2003 Asymmetric flows of viscoelastic fluids in symmetric planar expansion geometries. J. Non-Newt. Fluid Mech. 114, 33. (doi:10.1016/S0377-0257(03)00117-4.)

Phan-Thien, N. \& Tanner, R. I. 1977 A new constitutive equation derived from network theory. J. Non-Newt. Fluid Mech. 2, 353. (doi:10.1016/0377-0257(77)80021-9.)

Poole, R. J. \& Escudier, M. P. 2004 Turbulent flow of viscoelastic liquids through an axisymmetric sudden expansion. J. Non-Newt. Fluid Mech. 117, 25. (doi:10.1016/j.jnnfm.2003.11.007.)

Rodd, L. E., Scott, T. S., Cooper-White, J. J. \& McKinley, G. H. 2005 Capillary Break-up rheometry of low-viscosity elastic liquids. Appl. Rheol. 15, 12.

Schrek, E. \& Schafer, M. 2000 Numerical study of bifurcation in three-dimensional sudden channel expansions. Comput. Fluids 29, 583. (doi:10.1016/S0045-7930(99)00014-6.)

Schümmer, P. \& Tebel, K. H. 1983 A new elongational rheometer for polymer solutions. J. NonNewt. Fluid Mech. 12, 331.

Shah, R. K. \& London, A. L. 1978 Laminar flow forced convection in ducts. New York: Academic Press.

Stelter, M., Brenn, G., Yarin, A. C., Singh, R. P. \& Durst, F. 2000 Validation and application of a novel elongational device for polymer solutions. J. Rheol. 44, 595. (doi:10.1122/1.551102.)

Townsend, P. \& Walters, K. 1994 Expansion flows of non-Newtonian liquids. Chem. Engng Sci. 49, 749. (doi:10.1016/0009-2509(94)85020-8.)

Walters, K., Bhatti, A. Q. \& Mori, N. 1990 In The influence of polymer conformation on the rheological properties of aqueous polymer solutions (ed. D. De Kee \& P. N. Kaloni) Recent developments in structured continua, vol. 2. London: Pitman.

Wheeler, J. A. \& Whissler, E. H. 1966 Steady flow of non-Newtonian fluids in a square duct. Trans. Soc. Rheol. 10, 353. (doi:10.1122/1.549051.)

Yasuda, K., Armstrong, R. C. \& Cohen, R. E. 1981 Shear flow properties of concentrated solutions of linear and star branched polystyrenes. Rheol. Acta 20, 163. (doi:10.1007/BF01513059.) 